\title{
A mesomodel for the numerical simulation of the multiphasic behavior of materials under anisothermal loading (application to two low-carbon steels)
}

\author{
M. Coret ${ }^{\mathrm{a}}$, A. Combescure ${ }^{\mathrm{b}}$ \\ ${ }^{a}$ LMT-Cachan, E.N.S. Cachan/C.N.R.S. UMR 8535/Université Paris 6, 61, Avenue du Président Wilson, \\ 94235 Cachan Cedex, France \\ ${ }^{\mathrm{b}}$ LMC, UMR C.N.R.S./INSA 5514, 18-20 rue des sciences, 69621 Villeurbanne Cedex, France
}

The determination of residual stresses induced by welding or heat treatment operations requires the use of complex models taking into account thermal, metallurgical and mechanical phenomena. In this paper, we propose a mechanical model in which each phase can follow its own constitutive law. This model also takes into account phase transformation plasticity, which is treated independently of the behavior of each phase. This model has been implemented into the French FEM code Castem 2000. The interest of the proposed method is that it allows one to mix any type of nonlinear behavior using Taylor homogenization hypothesis. There is no need to develop a theory to get the equations of the homogenized material law. Two numerical examples demonstrate the efficiency and the flexibility of this approach. The results obtained are compared to experimental values for a typical welding situation and a high-temperature response. This comparison seems to indicate that viscous effects in the materials have a significative influence on the residual stresses produced by welding.

Keywords: Finite element calculation; Multiphase material; Transformation-induced plasticity; Residual stresses

\section{Introduction}

The performance in service of parts that have been subjected to welding or heat treatment depends on the residual stress state of the structure. Proper evaluation of these stresses is available only through calculation. However, the models involved in such calculations must take into account 


\begin{tabular}{|c|c|}
\hline \multicolumn{2}{|c|}{ Nomenclature } \\
\hline$E^{t}$ & total macroscopic strain \\
\hline$E^{e}$ & elastic macroscopic strain \\
\hline$E^{\text {thm }}$ & thermometallurgical macroscopic strain \\
\hline$E^{p}$ & plastic macroscopic strain \\
\hline$E^{t p}$ & phase transformation plastic strain \\
\hline$\varepsilon_{i}^{t}$ & total microscopic strain of the $i$ th phase \\
\hline$\varepsilon_{i}^{e}$ & elastic microscopic strain of the $i$ th phase \\
\hline$\varepsilon_{i}^{t h m}$ & thermometallurgical strain of the $i$ th phase \\
\hline$\varepsilon_{i}^{v p}$ & viscoplastic microscopic strain of the $i$ th phase \\
\hline$\Delta \varepsilon_{\alpha, \gamma}^{T_{r e f}}$ & difference of compactness of phases $\alpha$ compared to phases $\gamma$ at $T_{r e f}$ \\
\hline$\sum$ & macroscopic stress \\
\hline$S$ & deviator of macroscopic stresses \\
\hline$\Sigma^{y}$ & yield strength \\
\hline$h$ & macroscopic hardening coefficient \\
\hline$\sigma_{i}$ & microscopic stress of the $i$ th phase \\
\hline$s_{i}$ & deviator of microscopic stresses \\
\hline$\sigma_{i}^{y}$ & yield strength of the $i$ th phase \\
\hline$h_{i}$ & hardening coefficient of $i$ th phase \\
\hline$\sigma_{\gamma}$ & yield strength of austenite \\
\hline $\mathfrak{H}$ & Hooke's operator \\
\hline$X_{i}$ & kinematic strain hardening \\
\hline$R_{i}$ & isotropic strain hardening \\
\hline$\alpha_{i}$ & thermal expansion coefficient of the $i$ th phase \\
\hline$z_{i}$ & volume ratio of the $i$ th phase \\
\hline$z_{\gamma}$ & volume ratio of the austenitic phase \\
\hline$d$ & austenitic grain size \\
\hline$T$ & temperature \\
\hline$T_{r e f}$ & reference temperature \\
\hline$q$ & heat flux \\
\hline $\bar{\rho}$ & mass density \\
\hline$c$ & thermal capacity \\
\hline$l_{i}$ & latent heat of the $i$ th phase \\
\hline$k^{\prime}$ & thermal conductivity \\
\hline$r$ & heat input \\
\hline 1 & unit tensor \\
\hline
\end{tabular}

the structural transformations undergone by the parts. The modeling procedure proposed is applied to low-alloy ferritic steels such as 16MND5 or SA533, which are used in the manufacturing of pressurized water nuclear reactor vessels. Let us recall that phase transformations in low-carbon 


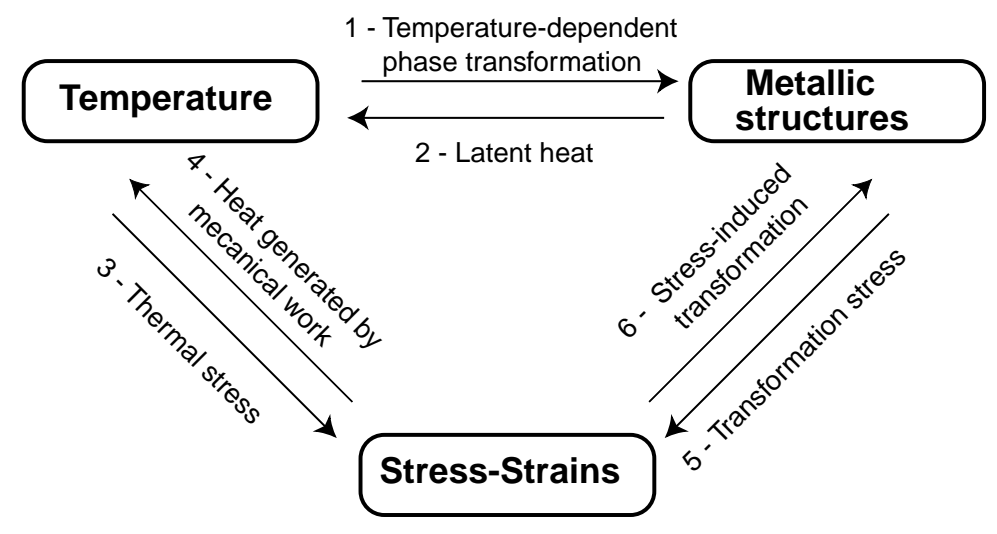

Fig. 1. Coupling mechanisms.

steels are predominant factors, along with thermal expansion, in the generation of residual stresses. These steel types have an austenitic structure at high temperature and a ferritic structure at ambient temperature. Let us recall that because these two phases have different densities, the transition from one to the other causes a volume variation (which we called $\Delta \varepsilon_{\alpha, \gamma}^{T_{r e f}}$ ). Moreover, depending on the cooling rate, a number of ferrites, with considerably different behavior, are likely to develop. Therefore, the material must be treated as a heterogeneous medium whose different constituents' characteristics depend greatly on the thermomechanical history.

The simulation of such problems must account for the various coupling mechanisms which exist among the different loads. A graphic representation of these coupling mechanisms (see Fig. 1) was given by Inoue [1]. Some authors [1-3] proposed macroscopic models to take all these phenomena into consideration in a common framework. In our approach, we consider the influences of mechanics on thermics (arrow no. 4) and of mechanics on metallurgy (arrow no. 6) as second-order effects, since it has been observed that for such steels the influence of the stress state on the transformation diagrams is small [4,5]. This assumption enables us to solve the thermometallurgical problem independently of the mechanical one. We rely on thermomechanics calculation and prediction methods which were described in detail in Refs. [6,7]. Our work focuses only on the simulation of the mechanical behavior given the thermometallurgical state of the material.

In the first part, we present the equations of the problem. A specific treatment is provided for the mechanical behavior of the multiphasic material. The second part is dedicated to the application of the model to two numerical simulation examples.

\section{Modeling}

\subsection{The thermometallurgical problem}

The first step in the calculation consists of determining the temperature $T$, the heat flux $q$ and the phase ratios $z_{i}$ at each point in the structure $\Omega$. The material is considered to be homogeneous with respect to the thermal characteristics and its behavior follows Fourier's law (Eq. (4)). Coefficients $c$, $k$ and $\rho$ depend only on the temperature. The coupling between thermics and metallurgy is reflected, 


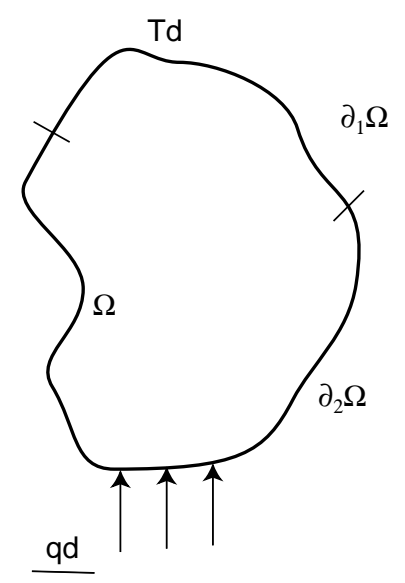

Fig. 2. Boundary conditions.

on the one hand, by the influence of the temperature and its derivative on the evolution of the phase ratios and, on the other hand, by the latent heat which coincides with phase transformations and is expressed by coefficients $l_{i}$.

Furthermore, $T d$ and $q d$ are the prescribed temperature and heat flux on the boundary of the structure (Eqs. (1) and ( $\overline{2)}$ ) (Fig. 2).

Boundary conditions: Imposed temperatures on $\partial_{1} \Omega$

$$
T=T d \text {. }
$$

Imposed heat flux on $\partial_{2} \Omega$

$$
q \underline{n}=\underline{q d} .
$$

Heat equation:

$$
\rho c \dot{T}=-\operatorname{div} \underline{q}-\rho \sum_{i=1}^{n} l_{i} \dot{z}_{i}+\rho r .
$$

Constitutive relation:

$$
\underline{q}=-k \underline{\operatorname{grad}}(T) .
$$

In calculating the phase ratios, we assume that their evolution is governed by $T, \dot{T}$ and $d$. Thus,

$$
\dot{z}_{i}=f\left(z_{i}, T, \dot{T}, d\right)
$$

and

$$
\sum_{i=1}^{n} z_{i}=1 .
$$

It is extremely difficult to present a unified formulation of all possible transformations in a low-carbon steel. There are different laws which apply either to the diffusion transformations [1,8-10] or to the martensitic transformation [11]. Here, we use a Waeckel's model [7], in which the evolution of the phase ratios is calculated from a continuous cooling transformation (CCT) diagram obtained 


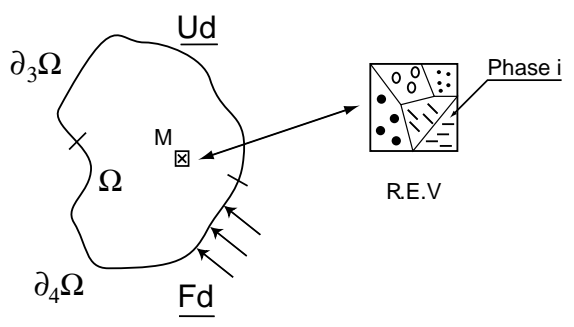

Fig. 3. Mechanical and R.E.V. boundary conditions.

experimentally. The evolution of the phases, generated by diffusion is obtained by linear interpolation in the CCT. For temperatures lower than the martensitic transformation temperature, the generation of martensite is governed by Koistinen-Marburger's law.

The thermometallurgical problem is solved using the finite element calculation program Castem 2000 [12], in which the thermometallurgical model was implemented by Martinez [6].

\subsection{The mechanical problem}

\subsubsection{Equilibrium equations}

In the second step of the calculation, given the temperature and phase ratio fields, we seek the stress $\Sigma$ and displacement $\underline{U} \cdot \underline{U d}$ and $\Sigma \underline{n}$ are the prescribed displacements and loads on the boundary of the structure Fig. 3.

Boundary conditions:

$$
\begin{array}{ll}
\underline{U}=\underline{U d} & \text { on } \partial_{3} \Omega, \\
\underline{F}=\Sigma \underline{n} & \text { on } \partial_{4} \Omega .
\end{array}
$$

Equilibrium equations:

$$
\underline{\operatorname{div}} \Sigma(M)=\underline{0} \quad \forall M \in \Omega .
$$

Constitutive relation: to be determined.

\subsubsection{Behavior of the multiphasic material}

The phases obtainable with a low-alloy steel such as 16MND5 or A533 (austenite, martensite, bainite or ferrite-perlite) are morphologically very different. Consequently, their mechanical properties are, of course, different. For example, austenite - which appears at high temperature-is by nature much more viscous than martensite. Thus, studying the macroscopic behavior becomes a homogenization problem which is extremely difficult to solve without making numerous assumptions.

Explicit models can do away with the behavior of the different phases by seeking a behavioral model only on the macroscopic scale. Thus, Inoue et al. [1], Hamata et al. [2] or Aliaga et al. [3] assume only internal variables on the macroscopic scale and infer the ratios of the different phases from an energy-based mixing law. The behavior can be plastic or viscoplastic and transformation plasticity is viewed as an additional dissipative term. The problem with such models is that they must be identified for all possible temperatures and phase ratios. Besides, all phases are assumed to have similar behavior. 
Another approach, called micro-macro, consists of starting from the behavior of each phase and working back to the macroscopic behavior of the material. Thus, Leblond et al. [13] showed, in the case of two elastic-plastic phases with strain hardening having the same yield strength, that the global behavior is also elastic-plastic with strain hardening. Furthermore, they gave a theoretical account of the source of the phase transformation plasticity and provided an expression for this quantity which is valid under a wide range of hypotheses. However, it seems rather difficult to extend this work to the case of $n$ elastic-viscoplastic phases.

(a) Homogenized macroscopic behavior. Therefore, we chose to group the models mentioned above under the term "mixing law" and we programmed the following model as denoted by the reference model.

We choose the following:

Partitioning of the macroscopic strain rates:

$$
\dot{E}^{t}=\dot{E}^{e}+\dot{E}^{t h m}+\dot{E}^{p}+\dot{E}^{t p} .
$$

The elastic macroscopic constitutive equations are

$$
E^{e}=\mathbb{U}^{-1}(T) \Sigma+\left[E^{t h m}(T)-E^{t h m}\left(T_{r e f}\right)\right]
$$

with

$$
E^{t h m}(T)=\sum_{i=1}^{n} z_{i} \alpha_{i}(T) \cdot T 1-\left(1-z_{\gamma}(T)\right) \Delta \varepsilon_{\alpha, \gamma}^{T_{r e f}},
$$

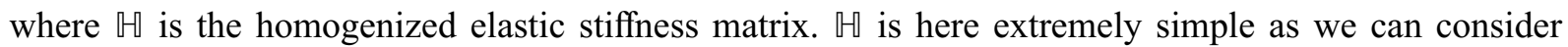
that all phases have the same Young's modulus and the same Poisson's ratio [1,6].

We use an usual elastoplastic model, with isotropic hardening, defined by

$$
f=J_{2}(S)-h-\Sigma^{y}\left(z_{i}\right)
$$

with

$$
\begin{aligned}
& J_{2}(S)=\left[\frac{3}{2} S: S\right]^{1 / 2}, \\
& \Sigma^{y}\left(z_{i}\right)=\sum_{i=1}^{n} z_{i} \sigma_{i}^{y} \text { and } h\left(z_{i}\right)=\sum_{i=1}^{n} z_{i} h_{i} .
\end{aligned}
$$

The associated law for definition of elastoplastic macroscopic strain rate is the usual one:

$$
\dot{E}^{p}=\frac{3}{2} \frac{S}{J_{2}(S)} \dot{\Lambda} .
$$

The phase strain rate is given by the Leblond [13] rate equations

$$
\dot{E}^{t p}= \begin{cases}0 & \text { if } z_{\gamma} \leqslant 0.03, \\ -3 \frac{\Delta \varepsilon_{\alpha, \gamma}^{T_{r e f}}}{\sigma_{\gamma}^{y}} \ln \left(z_{\gamma}\right) \dot{z}_{\gamma} S & \text { if } z_{\gamma}>0.03 .\end{cases}
$$

(b) Mesomodel. Our approach, which is much more numerically oriented, ignores an a priori constitutive law for each phase. As already noted, there seems to be no valid reason to use the 
same type of behavior for the austenitic and martensitic phases, for example. Furthermore, we do not always have, at our disposal, exhaustive test results for all phases. Indeed, identifications of viscoplastic models are more complex and more expensive than a simple elastic-plastic identification. The homogenizing procedure used is the following: Taylor's localization law [14] (which assumes homogeneous deformations in a heterogeneous medium with nonlinear behavior) is used, which provides the closest possible match with Leblond's theoretical case for elastic-plastic phases. Therefore, we split the total strain rate into two parts, one coming from the total microscopic strain rate of the phases and the other representing the plastic transformation strain rate. Thus

$$
\begin{aligned}
& \dot{E}^{t}=\dot{E}+\dot{E}^{t p}, \\
& \dot{E}=\varepsilon_{i} \quad \forall i .
\end{aligned}
$$

In this model, classical plasticity and transformation plasticity are assumed to be uncoupled, which is true for small strains. Thus, the homogenization law for stresses is

$$
\Sigma=\sum_{i=1}^{n} z_{i} \sigma_{i} .
$$

This modeling scheme provides great flexibility in the calculation. Arbitrary constitutive laws, as well as different models of transformation plasticity rates, can be selected for each phase.

We now describe the equation of the mesomodel.

We partition the strains using

$$
\dot{E}^{t}=\dot{\varepsilon}_{i}+\dot{E}^{t p} \quad \forall i
$$

and

$$
\dot{\varepsilon}_{i}=\dot{\varepsilon}_{i}^{e}+\dot{\varepsilon}_{i}^{t h m}+\dot{\varepsilon}_{i}^{v p} \quad \forall i
$$

The transformation plasticity strain rate is given by [13]

$$
\dot{E}^{t p}= \begin{cases}0 & \text { if } z_{\gamma} \leqslant 0.03, \\ -3 \frac{\Delta \varepsilon_{\alpha, \gamma}^{T_{r e f}}}{\sigma_{\gamma}^{y}} \ln \left(z_{\gamma}\right) \dot{z}_{\gamma} S & \text { if } z_{\gamma}>0.03 .\end{cases}
$$

For the behavior of phases, various behavioral models were tested. Elastic and thermometallurgical strains are the same for all models.

$$
\varepsilon_{i}^{e}=\sharp^{-1}(T) \sigma_{i}+\left[\varepsilon_{i}^{t h m}(T)-\varepsilon_{i}^{t h m}\left(T_{r e f}\right)\right]
$$

with

$$
\varepsilon_{i}^{t h m}(T)=\alpha_{i}(T) \cdot T 1 \quad \text { for the ferritic phases }
$$

and

$$
\varepsilon_{i}^{t h m}(T)=\alpha_{i}(T) \cdot T 1-\left(1-z_{\gamma}(T)\right) \Delta \varepsilon_{\alpha, \gamma}^{T_{r e f}} \quad \text { for austenite. }
$$


The nonlinear characteristics chosen are explained now:

- For the martensite, elastic-plastic model with kinematic strain hardening is chosen.

$$
\begin{aligned}
f & =J_{2}\left(s_{i}-X_{i}\right)-\sigma_{i}^{y}, \\
\dot{\varepsilon}_{i}^{p} & =\frac{3}{2} \frac{\left(s_{i}-X_{i}\right)}{J_{2}\left(s_{i}-X_{i}\right)}, \\
X_{i} & =\frac{2}{3} H \varepsilon_{i}^{p} .
\end{aligned}
$$

We choose an elastoplastic behavior because the martensite appears at low temperature.

- For bainite and ferrite, we have used either elastic-plastic (Eqs. (26)-(28)) or Chaboche [15] elastic-viscoplastic models. This model includes two kinematic hardening and one isotropic hardening mechanisms.

$$
\begin{aligned}
& f=J_{2}\left(s_{i}-X_{i}\right)-R_{i}-\sigma_{i}^{y}, \\
& \dot{\varepsilon}_{i}^{p}=\frac{3}{2} \frac{\left(s_{i}-X_{i}\right)}{J_{2}\left(s_{i}-X_{i}\right)}, \\
& \dot{p}_{i}=\left\langle\frac{J_{2}\left(s_{i}-X_{i}\right)-R_{i}-\sigma_{i}^{y}}{K_{i}}\right\rangle^{n}, \\
& \dot{R}_{i}=b_{i}\left(Q_{i}-R_{i}\right) \dot{p}_{i} \quad R_{i}(0)=0, \\
& X_{i}=X_{1 i}+X_{2 i}, \\
& X_{1 i}=C_{1} \alpha_{1 i} \quad \dot{\alpha}_{1 i}=\dot{\varepsilon}_{i}^{v p}-D_{1 i} \alpha_{1 i} \dot{p}_{i} \quad \alpha_{1 i}(0)=0, \\
& X_{2 i}=C_{2} \alpha_{2 i} \quad \dot{\alpha}_{2 i}=\dot{\varepsilon}_{i}^{p} \quad \alpha_{2 i}(0)=0 .
\end{aligned}
$$

Bainite and ferrite appear at rather high temperature $\left(600^{\circ} \mathrm{C}\right)$ for slow cooling rates. We have chosen to modelize their behavior either with elastoplastic or with viscoplastic in order to see the influence of the viscosity on the residual stresses.

- For austenite, we have used either elastic-plastic (Eqs. (26)-(28)) or Chaboche elastic-viscoplastic models for 16MND5 (Eqs. (29)-(35)) or Norton-Bailey creep model for SA533. Equations of the Norton-Bailey model are

$$
\begin{aligned}
& f=J_{2}\left(s_{i}\right)-\sigma_{i}^{y}, \\
& \dot{\varepsilon}_{i}^{v p}=\dot{p}_{i} \frac{3}{2} \frac{s_{i}}{J_{2}\left(s_{i}\right)}, \\
& p_{i}=A(T) J_{2}\left(s_{i}\right)^{m(T)} t^{n(T)} .
\end{aligned}
$$

Austenite appears at high temperature, we have then chosen a viscous model for this phase. For the application to the 16MND5 material, an identification was available from the work of Martinez [6]. For the application to SA533, the identification was available with a Norton's law from US data base [16]. 

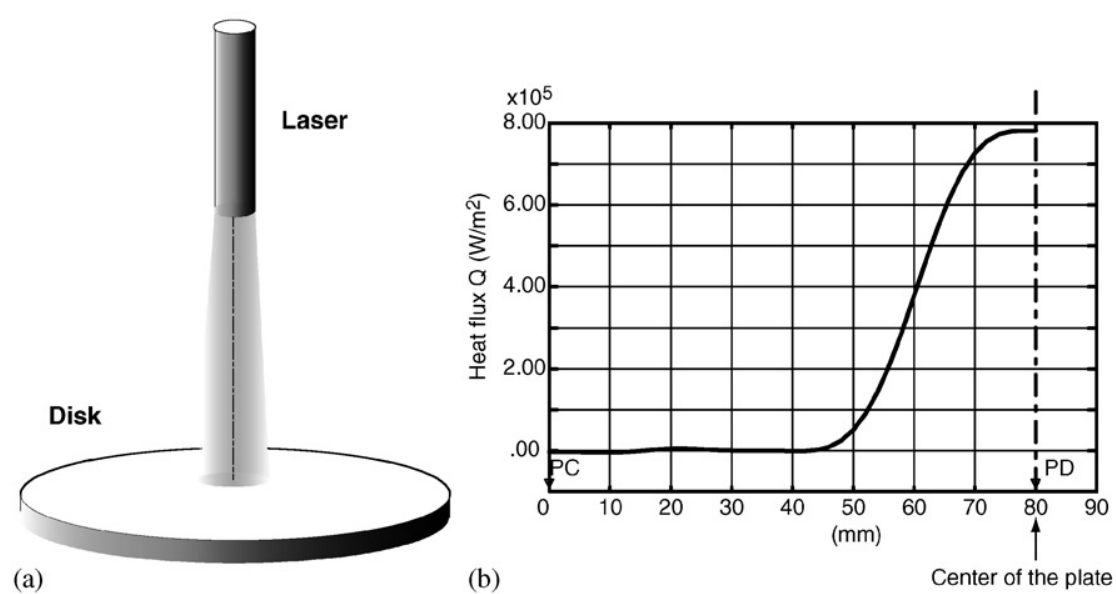

Fig. 4. Laser heating: (a) laser-heated disk; and (b) prescribed heat flux.

\subsubsection{Integration of the equations in the time domain}

The equations of the behavioral model are expressed in terms of strain rates; therefore, they must be integrated over time in order to obtain strains. Usually, explicit or implicit Euler-type methods are used. We use an implicit formulation in which the temperature-dependent material properties are assumed to remain constant during the integration step and equal to their values at the end of the step.

\section{Applications}

All model characteristics are given in Appendix A

\subsection{Simulation of a laser-heated disk}

This first simulation reproduces a test made at INSA de Lyon [17]. Its purpose was to understand and analyze the residual stresses produced during a welding operation. A disk made of 16MND5, $160 \mathrm{~mm}$ in diameter and $8 \mathrm{~mm}$ thick, was heated at the center by a spot laser for $70 \mathrm{~s}$, then cooled by natural convection (Fig. 4(a)). The temperatures at the underside of the disk were measured along with the displacement at the center. At the end of the test, the residual stresses were measured by X-ray diffraction.

The numerical simulation was performed with the analysis program Castem 2000. The mesh consisted of 320 QUA4 elements and the problem was considered axisymmetric.

\subsubsection{Operating sequence of the thermometallurgical calculation}

The spot laser was modeled by a heat flux whose distribution on the upper side is known (Fig. 4(b)). The lower and lateral sides were subjected to free convection. Finally, initial structure of the disk was completely bainitic. One can see that at the end of the heating stage (Fig. 5(a)), only the central part of the disk reached a temperature higher than $700^{\circ} \mathrm{C}$, which is the starting temperature of austenitic transformation. Since cooling occurred very rapidly, this austenite produced 


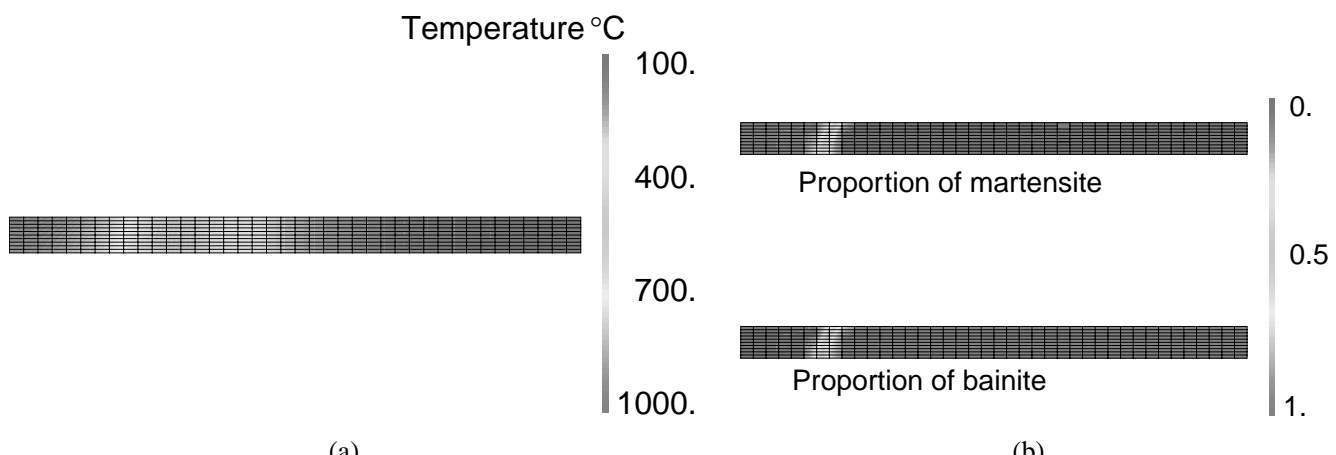

(a)

(b)

Fig. 5. Phase ratio field and temperature field: (a) temperature at the end of the heating stage; and (b) phase ratios at the end of the test.
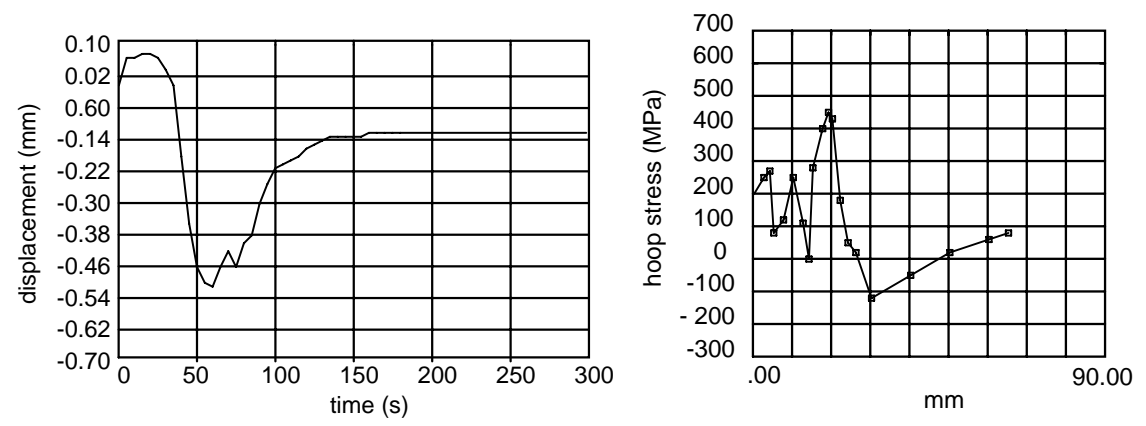

Fig. 6. Displacement at the center and hoop stress on the upper side-experimental values.

mostly martensite at the end of the cooling stage. The computed distributions are represented in Fig. 5(b). These computed fields are in very good agreement with experimental observations in terms of metallurgical composition as well as geometrical position [17].

\subsubsection{Operating sequence of the mechanical calculation}

The mechanical loading consisted of prescribing the temperatures and phase ratios previously calculated. The time discretization can be different for the thermometallurgical and for the mechanical calculations. If this is the case, the loading values are interpolated linearly. Various simulations were performed: the first one used the mixing law model in which the different phases are elastic-plastic (Figs. 6 and 7); the next two simulations were performed with the mesomodel. In the first case, all phases were elastic-plastic with strain hardening (Fig. 8), whereas in the second case the martensite was elastic-plastic with strain hardening and the other phases were viscoplastic (Fig. 9). We first note that the mesomodel with elastic-plastic phases and the mixing law model give very similar results both in terms of displacements and in terms of stresses. However, the response with these two models does not match the experimental results of Fig. 6 very well. These simulations produce stresses which are too high and displacements which are too large. Further, the evolution of the displacement at the beginning of the heating stage matches the measured data quite poorly. The results obtained with the mesomodel, using viscoplastic constitutive laws (Fig. 9) are much closer to the experimental results. 

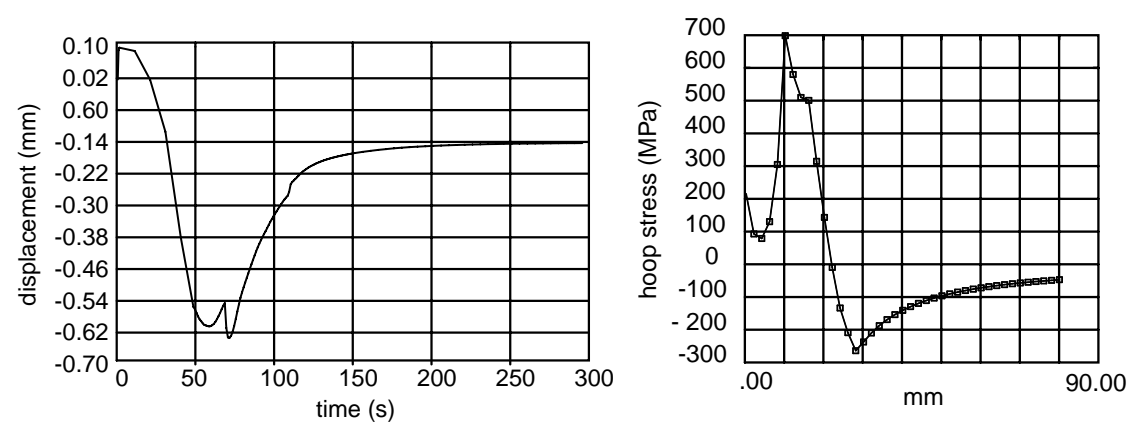

Fig. 7. Displacement at the center and hoop stress on the upper side-macroscopic elastic-plastic model.
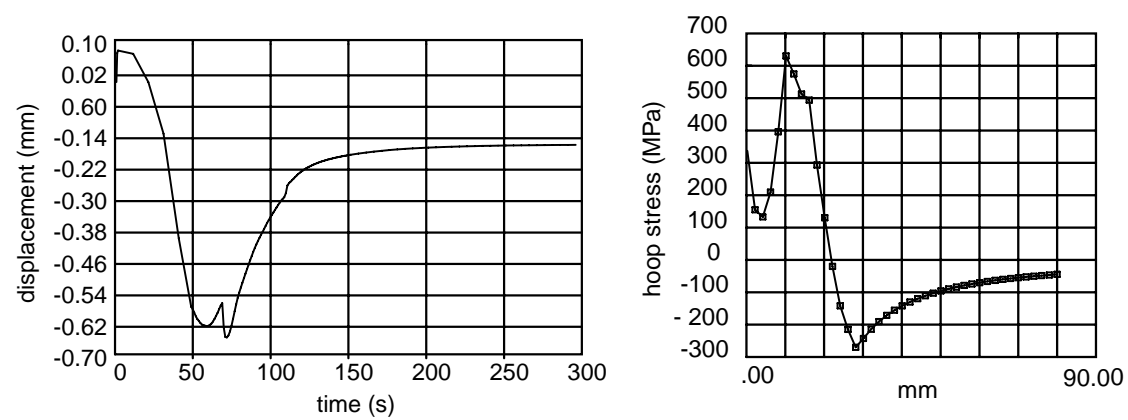

Fig. 8. Displacement at the center and hoop stress on the upper side-mesomodel with elastic-plastic phases.
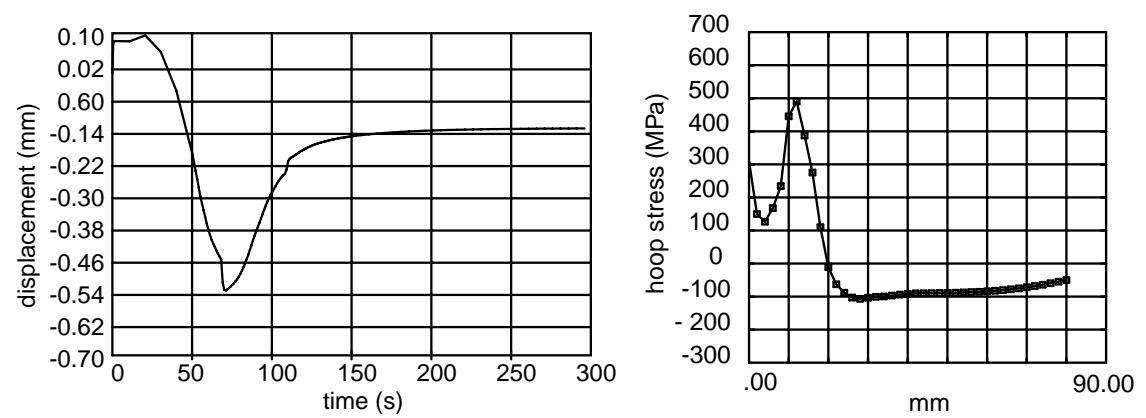

Fig. 9. Displacement at the center and hoop stress on the upper side-mesomodel with elastic-plastic and viscoplastic phases.

The residual stress level is good and the evolution of the displacement matches the measured data. The main difference between these simulations is viscosity. This parameter lowers the residual stress level and softens the structure at high temperature.

\subsection{Simulation of the rupture of a vessel heated under internal pressure}

The second example proposed is the simulation of a test carried out at Sandia National Laboratory on a scale $1 / 4.85$ nuclear reactor vessel subjected to low internal pressure and a $200^{\circ} \mathrm{C}$ temperature 

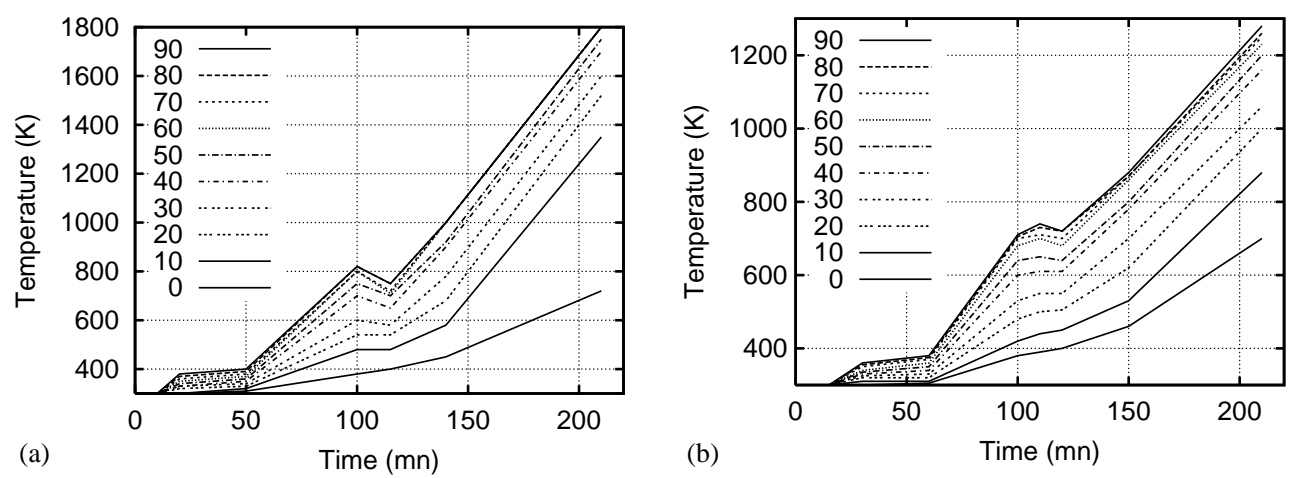

Fig. 10. Prescribed temperatures: (a) inner temperature; and (b) outer temperature.

gradient through the thickness. This type of simulation is particularly important in order to predict the behavior of the vessel during an emergency procedure following fusion of the reactor's core. The miniature SA533B1 steel vessel had an interior diameter of $91.4 \mathrm{~cm}$ and a thickness of $7.62 \mathrm{~cm}$. The spherical part was welded to a $61 \mathrm{~cm}$ high cylinder closed by a heavy cap bolted along the circumference. A susceptor heated by a $750 \mathrm{~kW}$ inductor was used to raise the vessel's temperature at a rate of $12^{\circ} \mathrm{Cmn}^{-1}$, to an inside temperature of $1500^{\circ} \mathrm{C}$. The numerical simulation was performed with a mesh made of 100 QUA4 elements and the problem was considered axisymmetric.

\subsubsection{Operating sequence of the thermometallurgical calculation}

The temperatures at the inner and outer faces were prescribed and equal to those measured during the test. Fig. 10(a) and (b) describe the measured temperatures on the inner and outer surface of the shell at angles $0-90^{\circ}$, where $0^{\circ}$ is the pole of the hemisphere. The thermal characteristics $(c, k)$ were those of US steel except that, in the absence of information on the CCT diagram for this steel, we used the one for 16MND5, which seemed reasonable considering how close these steel grades are. The initial structural content of the vessel was purely bainitic. The temperature field and the austenitic phase ratio field are plotted in Fig. 11(a) and (b). Let us observe that at time $t=170 \mathrm{mn}$ the austenitic transformation front had not gone completely through the wall yet, which is very important for the strength of the vessel because austenite is much more viscous than bainite. The maximum temperature at the end of the test was on the order of $1500^{\circ} \mathrm{C}$ inside the vessel.

\subsubsection{Operating sequence of the mechanical calculation}

The prescribed pressure through the calculation was the pressure measured during the test, i.e. 5.25 $\mathrm{MPa}$. The behavior of the bainite was considered to be elastic-viscoplastic according to Chaboche's model whereas the behavior of austenite followed a Norton model. Moreover, the transformation plasticity term was also included during the heating stage using Leblond's law. The results of the mechanical calculation are shown in Fig. 11(c) and (d). One can observe a relatively complex stress state resulting from the temperature gradient and the phase change with volume variation. Given the stress level reached, the austenite appears completely plastic. Therefore, rupture occurs rapidly by viscoplastic collapse once the transformation proceeds throughout the thickness. We note the good quality of the displacements predicted from the calculation, which come very close to the measured values. 


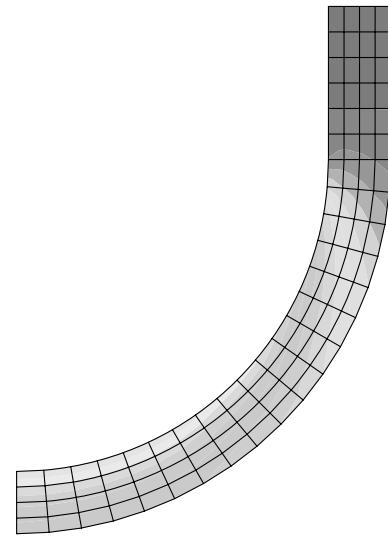

(a)

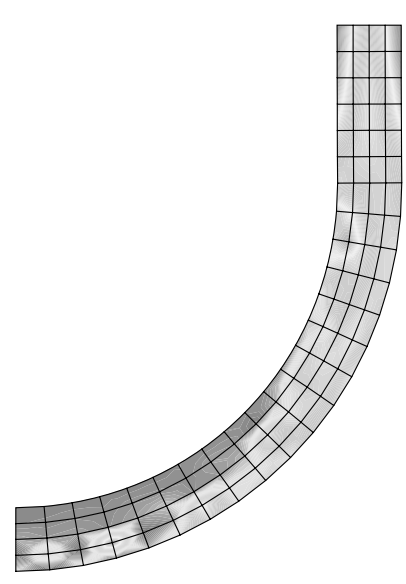

(c)

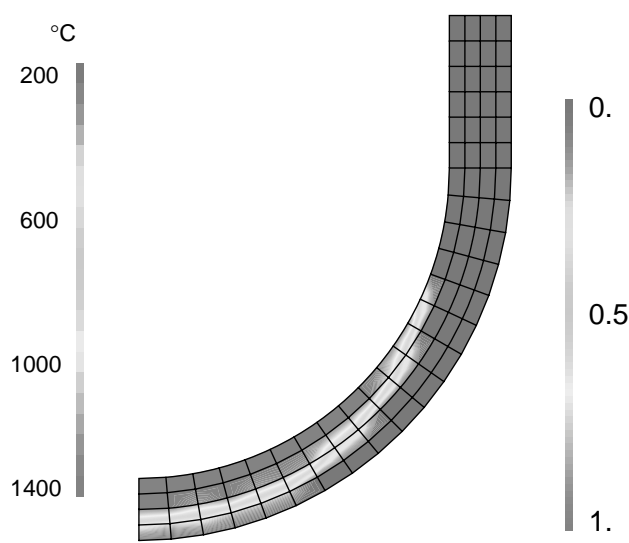

(b)

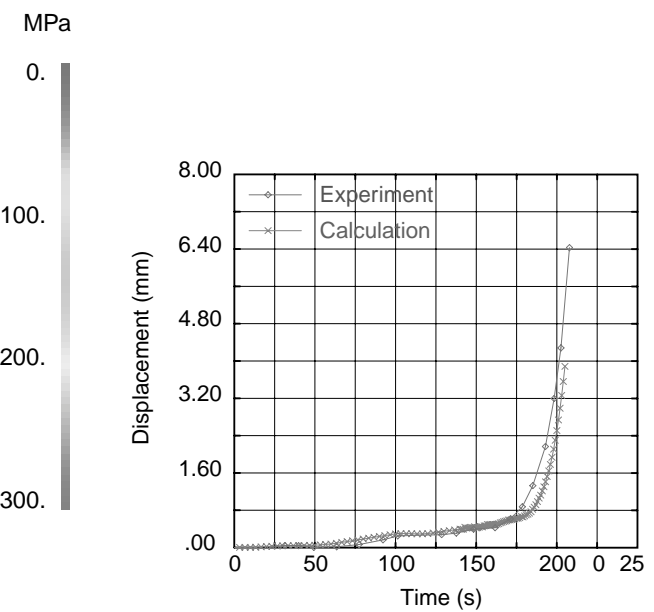

(d)

Fig. 11. Simulation of OLHF2: (a) temperatures $(t=170 \mathrm{mn})$; (b) Austenitic phase ratio $(t=170 \mathrm{mn})$; (c) Von Mises' stresses $(t=170 \mathrm{mn})$; and (d) vertical displacement at the vessel's center.

\section{Conclusion}

The model proposed takes into account the thermal, metallurgical and mechanical phenomena which occur during the simulation of welding or quenching operations. The phase ratios are predicted based on a CCT diagram. The mechanical calculation, which includes the difference in phase compactness and transformation plasticity, takes into account the actual behavior of each phase. The consistency of the model is demonstrated by the good correlation between simulations and experimental results. Moreover the proposed two scale model allows one to make a numerical homogenization through Taylor's approximation. This avoids the theoretical development of a homogenization law. The price to pay is that we have to keep track of the material state of each phase during the whole process. This can also have some interest if one wants to take into account history effects. 
There is also no coup ling between elastoviscop lasticity and transformation plasticity. The numerical homogenization has the limitation in giving any exp licit equation for the homogenized material.

\title{
Acknowledgements
}

We express our deepest thanks to CEA/DMT which sponsored this work, provided the material and gave constant encouragement throughout our research.

\section{Appendix A. Constants and coefficients}

\author{
A.1. Metallurgy (see Fig. 12)
}

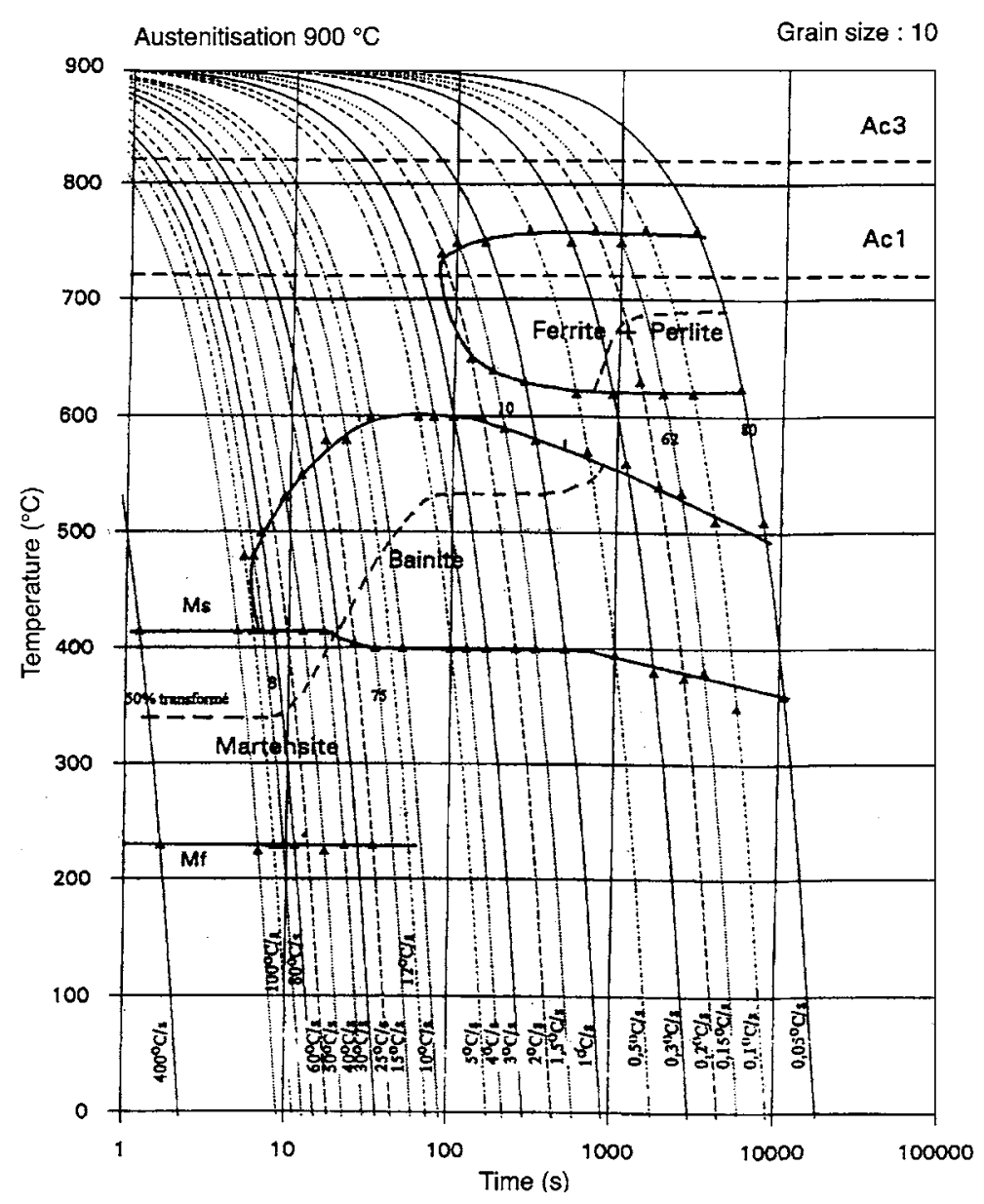

Fig. 12. CCT diagram of 16MND5 [15]. 


\section{A.2. Thermics (see Table 1)}

Table 1

Thermal characteristics [18]

\begin{tabular}{lcccccccc}
\hline$T\left({ }^{\circ} \mathrm{C}\right)$ & 20 & 200 & 300 & 400 & 600 & 750 & 900 & 1000 \\
\hline$\rho c\left(10^{6} \mathrm{~J} / \mathrm{m}^{3} \mathrm{~K}\right)$ & 3.49 & 4.09 & 4.42 & 4.80 & 6.1 & 10.13 & 5.88 & 4.76 \\
$k(\mathrm{~W} / \mathrm{mK})$ & 37.7 & 40.5 & 39.5 & 37.7 & 33 & 29.3 & 25.3 & 26.9 \\
\hline
\end{tabular}

\section{A.3. Mechanics}

Expansion coefficient:

ferritic phases: $\alpha_{\alpha}=15 \times 10^{-6^{\circ}} \mathrm{C}^{-1}$

austenitic phase: $\alpha_{\gamma}=23.5 \times 10^{-6^{\circ}} \mathrm{C}^{-1}$

Difference in phase compactness: $\Delta \varepsilon_{\alpha, \gamma}^{T_{r e f}}=1.1 e^{-2}$.

A.3.1. Elastic-perfectly plastic model and elastic-plastic model with strain hardening (see Table 2)

Table 2

Characteristics of the elastic-plastic models [6]

\begin{tabular}{|c|c|c|c|c|c|c|c|c|c|}
\hline$T\left({ }^{\circ} \mathrm{C}\right)$ & 0. & 100 & 200 & 400 & 600 & 700 & 800 & 900 & 1000 \\
\hline$E(\mathrm{Gpa})$ & 208 & 204 & 200 & 180 & 135 & 80 & 50 & 32 & 30 \\
\hline$\sigma_{y m}(\mathrm{MPa})$ & 1200 & 1170 & 1100 & 980 & 680 & 350 & 100 & 50 & 20 \\
\hline$\sigma_{y b}(\mathrm{MPa})$ & 480 & 450 & 430 & 390 & 270 & 140 & 70 & 30 & 20 \\
\hline$\sigma_{y f}(\mathrm{MPa})$ & 320 & 300 & 275 & 230 & 170 & 100 & 60 & 30 & 20 \\
\hline$\sigma_{y a}(\mathrm{MPa})$ & 140 & 130 & 120 & 110 & 100 & 70 & 60 & 30 & 20 \\
\hline$H_{m}(\mathrm{MPa})$ & 10,000 & 10,000 & 10,000 & 10,000 & 9000 & 8000 & 7000 & 6000 & 5000 \\
\hline
\end{tabular}

$m=$ martensite, $b=$ bainite, $f=$ ferrite-perlite, $a=$ austenite.

\section{A.3.2. Chaboche's elastic-viscoplastic model (see Tables 3 and 4)}

Table 3

Characteristics of the viscoplastic model for ferrite and bainite [6]

\begin{tabular}{rlllllrlrr}
\hline$T\left({ }^{\circ} \mathrm{C}\right)$ & $\begin{array}{l}\sigma_{y b} \\
(\mathrm{MPa})\end{array}$ & $\begin{array}{l}\sigma_{y f} \\
(\mathrm{MPa})\end{array}$ & $\begin{array}{l}Q \\
(\mathrm{MPa})\end{array}$ & $b$ & $\begin{array}{l}C_{1} \\
(\mathrm{MPa})\end{array}$ & $D_{1}$ & \multicolumn{1}{l}{$\begin{array}{l}C_{2} \\
(\mathrm{MPa})\end{array}$} & $\begin{array}{l}K \\
\left(\mathrm{MPa} \mathrm{s} \mathbf{s}^{1 / n}\right)\end{array}$ \\
\hline 20 & 254 & 200 & 65 & 0.38 & 349,000 & 960 & 4220 & 30 & 10 \\
300 & 254 & 200 & 65 & 0.38 & 349,000 & 960 & 4220 & 30 & 10 \\
550 & 15 & 15 & 4 & 2 & 154,600 & 970 & 2950 & 1140 \\
650 & 2.1 & 2.1 & 0 & 2 & 40,587 & 970 & 500 & 1112 & 5.72 \\
\hline
\end{tabular}


Table 4

Characteristics of the viscoplastic model for austenite (16MND5 steel) [6]

\begin{tabular}{|c|c|c|c|c|c|c|c|c|}
\hline$T\left({ }^{\circ} \mathrm{C}\right)$ & $\begin{array}{l}\sigma_{y a} \\
(\mathrm{MPa})\end{array}$ & $\begin{array}{l}Q \\
(\mathrm{MPa})\end{array}$ & $b$ & $\begin{array}{l}C_{1} \\
(\mathrm{MPa})\end{array}$ & $D_{1}$ & $\begin{array}{l}C_{2} \\
(\mathrm{MPa})\end{array}$ & $\begin{array}{l}K \\
\left(\mathrm{MPa} \mathrm{s}^{1 / n}\right)\end{array}$ & $n$ \\
\hline 300 & 110 & 65 & 0.38 & 67,200 & 960 & 1400 & 30 & 10 \\
\hline 550 & 15 & 0 & 0.38 & 48,500 & 970 & 1000 & 390 & 6.72 \\
\hline 650 & 2.1 & 0 & 0.38 & 38,800 & 970 & 500 & 600 & 5.68 \\
\hline 750 & 1 & 0 & 0.38 & 29,100 & 970 & 470 & 820 & 4.75 \\
\hline 900 & 0.33 & 0 & 0.38 & 1570 & 970 & 390 & 770 & 2.97 \\
\hline 1000 & 0.31 & 0 & 0.38 & 0 & 970 & 264 & 472 & 2.95 \\
\hline 1100 & 0.29 & 0 & 0.38 & 0 & 970 & 100 & 234 & 2.86 \\
\hline
\end{tabular}

\section{A.3.3. Norton-Bailey model for US steel (see Table 5)}

Table 5

Characteristics of the creep model for austenite (AS533 steel) [16]

\begin{tabular}{lllllllll}
\hline$T(\mathrm{C})$ & 0 & 300 & 550 & 650 & 750 & 900 & 1000 & 1300 \\
\hline$m$ & 4.22 & 4.22 & 4.22 & 4.22 & 4.22 & 3.67 & 3.67 & 3.67 \\
$n$ & 1 & 1 & 1 & 1 & 1 & 1 & 1 & 1 \\
$A(\mathrm{MPa} \mathrm{s})$ & $8.27 e^{-27}$ & $4.78 e^{-20}$ & $1.01 e^{-16}$ & $1.03 e^{-14}$ & $1.42 e^{-12}$ & $2.36 e^{-10}$ & $1.22 e^{-9}$ & $7.36 e^{-8}$ \\
\hline
\end{tabular}

\section{References}

[1] Inoue T, Wang Z. Coupling between stress, temperature, and metallic structures during processes involving phases transformations. Materials Science and Technology 1985;1:845-50.

[2] Hamata N, Billardon R, Marquis D, Ben Cheikh A. A model for nodular graphite cast iron coupling anisothermal elasto-viscoplasticity and phase transformation. In: Desai CS et al., editors. Constitutive laws for engineering materials. New York: ASME Press, 1991. p. 593-6.

[3] Aliaga C, Massoni E, Louin JC, Denis S. 3d finite element simulation of residual stresses and distortions of cooling workpieces. In: Third International Conference on Quenching and Control of Distortion. Prague, Czech Republic, March, 1999.

[4] Denis S, Gautier E, Beck G, Simon A. Stress-phase-transformation interaction, basics principle, modelling, and calculation of internal stresses. Materials Science and Technology 1985;1:805-14.

[5] Denis S, Gautier E, Sjöström S. Influence of stresses on the kinetics of pearlitic transformation during continuous cooling. Acta Metallurgica 1987;35:1621-32.

[6] Martinez M. Jonction 16MND5-INCONEL 690-316LN par soudage diffusion elaboration et calcul des contraintes résiduelles de procédé. PhD thesis, ENSMP, décembre, 1999.

[7] Waeckel F. Une loi de comportement thermo-métallurgique des aciers pour le calcul mécanique des structures. Thèse, ENSAM, Mars, 1994.

[8] Avrami M. Kinetics of phase change I: general theory. Journal of Chemical Physics 1939;7:103-12.

[9] Johnson WA, Mehl RF. Reaction kinetics in process of nucleation and growth. Transactions of the AIME $1939 ; 135: 416-45$.

[10] Fernandes FBM, Denis S, Simon A. Mathematical model coupling phase transformation and temperature evolution during quenching of steels. Materials Science and Technology 1985;1(10):838-44. 
[11] Koïstinen DP, Marbürger RE. A general equation prescribing extent of austenite-martensite transformation in pure Fe-C alloys and plain carbon steels. Acta Metallurgica 1959;7:59-60.

[12] Verpeaux P, Charras T, Millard A. CASTEM 2000: une approche moderne du calcul des structures, In: Fouet J-M, Ladevèze P, Ohayon R. editors. Calcul des Structures et Intelligence Artificielle, vol. 2. Pluralis, 1988. p. $261-71$.

[13] Leblond JB, Devaux J, Devaux JC. Mathematical modelling of transformation plasticity in steel I. Case of ideal-plastic phases. International Journal of Plasticity 1989;5:551-72.

[14] Taylor GI. Plastic strains in metals. Journal of the Institute of Metals 1938;62:307-24.

[15] Lemaitre J, Chaboche JL. Mécanique des matériaux solides, 2iéme ed. Paris: Dunod, 1996.

[16] Benoit C, Combescure A. etude thermométallurgique mécanique sur des cuves de réacteurs PWR application à un scénario d'accident grave et interprétation d'essais. Rapport interne n 240. LMT-Cachan, September, 2000.

[17] Cavallo N. Contribution à la validation expérimentale de modèles décrivant la ZAT lors d'une opération de soudage. Thèse, INSA Lyon, Avril, 1998.

[18] Dupas et P, Waekael F. Recueil bibliographique de caractérisation thermomécanique pour l'acier de cuve, les revêtements inoxydables et les alliages 182 et 600. Note Technique HI-74/93/097 HT-26/93/058/A, EDF, 1994. 